\title{
The Application of X-ray Micro- and Nano- Scale Computed Tomography to the Morphological and In-situ Dynamic Study of Polymer Foam Materials.
}

\author{
Brian M. Patterson ${ }^{1}$, Nikolaus L. Cordes ${ }^{1}$, Robert Gilbertson ${ }^{1}$, Zachary Smith ${ }^{1}$ \\ ${ }^{1}$ Los Alamos National Laboratory, Materials Science and Technology Division, Los Alamos, USA.
}

Polymer foam materials are ubiquitous in our modern society as both natural and man-made materials. These materials are used in a variety of applications including structural materials, thermal insulation, mechanical padding and many others. To even begin to understand their structure-property relationships, a good understanding of their structural morphology is needed. This presentation will focus on the measurement and analysis of the morphological statistics coupled with in-situ stress (compression/tension) of these foams in 3D using micro- and nano- scale X-ray computed tomography $(\mu \mathrm{CT}$, nanoCT) which is critical in understanding the bulk performance of these materials.

Coupling 3D data sets to a data analysis software package allows materials researchers to quantifiably compare lot-to-lot, formulation-to-formulation variances as well as collect in-situ performance metrics in ways never before possible[1,2]. When measured with CT, the heterogeneity of these foam materials in void sizes and their distributions within the sample become readily apparent. Measuring polymer foam test blocks in multiple locations shows that even the most basic measure (eg. \% void volume) can change greatly within a single, highly heterogeneous sample. Comparing shape molded parts with a test block, shows that the test block does not provide an accurate fiducial for the morphological characteristics. We will show the results of the examination of the in-situ compression performance of poly(dimethyl siloxane) (silicone) and a comparison of as-molded and test blocks of poly(urethane) (PU) foams.

Finding identical replacement materials for no-longer available formulations of PDMS foams is a difficult prospect. 25-year-old samples of the original formulation, known as S5370, from Dow-Corning are compared to de-formulated and modified formulations in $3 \mathrm{D}$ and quantifiably measured for morphological and compression performance behaviours. X-ray $\mu \mathrm{CT}$ is used to compare the structural (Figure 1a), morphological (Figure 1b) and Poisson ratio measurements (Figure 2). Modified formulations of PDMS foams with higher crosslinking are found to have smaller average void sizes but exhibit higher Poisson ratio, independent of \% void volume [2]. These materials have a relatively wide void size distribution, also independent of location within the thickness of the sample.

In contrast, PU foams, cross section shown in Figure 3a, have an average void size with a relatively narrow void size distribution (Figure 3b). For these foams, the average size decreases (and a decreasing $\%$ void volume) nearer the surface of the moulded part. Interestingly, the void shape in a test block are very uniform and highly spherical, but in a moulded part(especially in narrow necks in regions of higher laminar flow) the voids become more eccentric and show the direction of the flow (Figure $3 \mathrm{c}$ ).

References:

[1] BM Patterson, CE Hamilton (2010) Analytical Chemistry 82: 8537. Doi:10.1021/ac101522q

[2] BM Patterson, K Henderson, Z Smith (2013) Journal of Materials Science 48: 1986.

[3] LJ Gibson, MF Ashby (1997) Cellular Solids: Structure and Properties. Cambridge University Press, Cambridge 


\section{A}
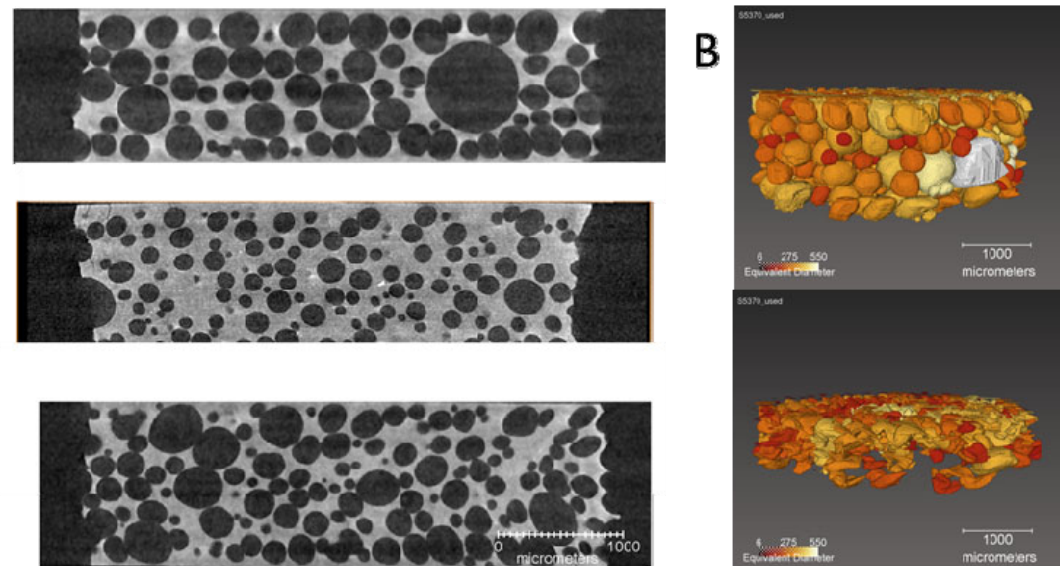

Figure 1. A) Reconstructed slices through S5370, SX462, and LK3626 formulations of silicone foams. Figure $1 \mathrm{~b}$ is a $3 \mathrm{D}$ rendering of $\mathrm{S} 5770$ foam at $0 \%$ (top) and $50 \%$ (bottom) compression. The voids are colored by equivalent diameter with sizes ranging up to $550 \mu \mathrm{m}$ diameter.

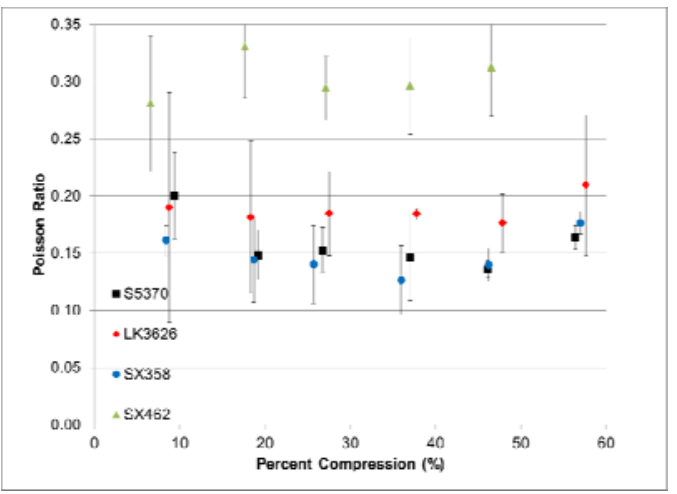

Figure 2. Measure of the Poisson ratio for four different formulations of silicone foams. Typical cellular solids have a Poisson ratio of $\sim 0.33$ [3]. Error bars are the standard deviations of three samples. The foam formulation with the highest Poisson ratio, SX462 also has the smallest average void size.

A

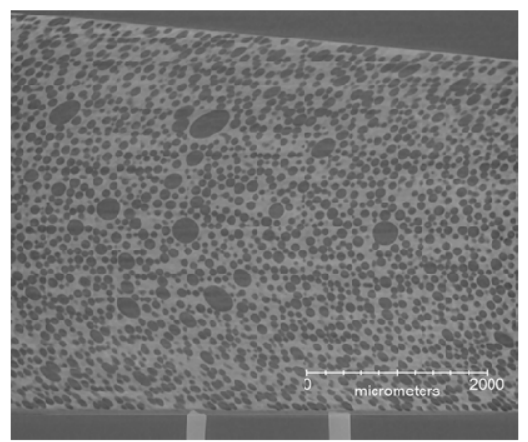

B

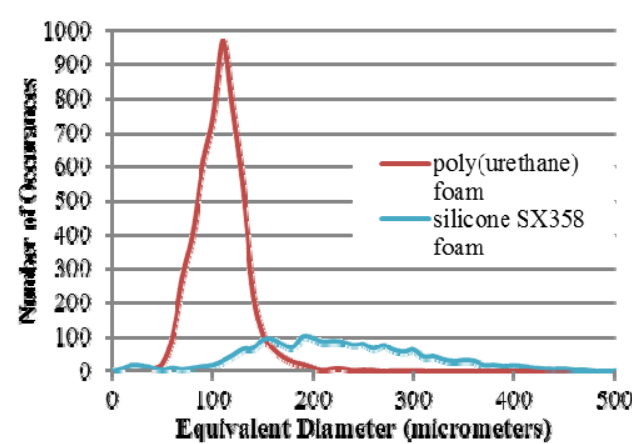

C

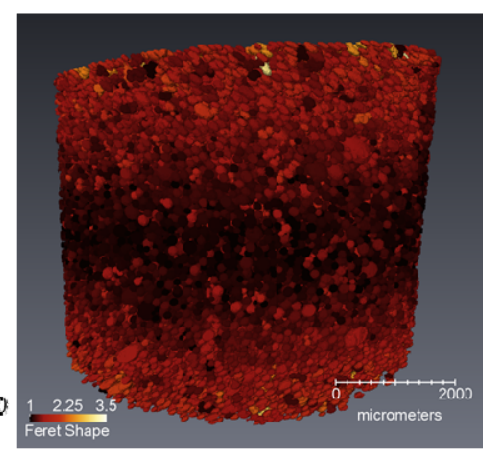

Figure 3. A) Reconstructed slice through the cross section of a sample of poly(urethane) foam. B) comparison of the equivalent diameter of the poly(urethane) (red) and silicone (blue). The poly(urethane) foam has a much narrower and well defined void size distribution. C) 3D rendering of the poly(urethane) foam voids exhibit an eccentricity closer to the surface due to laminar flow as a result of the blowing process. Coloring the voids by Feret shape, seen here, graphically demonstrates that phenomenon. Darker voids are more spherical, lighter voids are more oval. 